\title{
Theoretical and experimental studies of hyperreflective polymer-network cholesteric liquid crystal structures with helicity inversion
}

\author{
A. C. Tasolamprou ${ }^{\mathrm{a}}$, M. Mitov ${ }^{\mathrm{b}}$, D. C. Zografopoulos ${ }^{\mathrm{a}}$, and \\ E. E. Kriezis ${ }^{a}$ \\ ${ }^{a}$ Department of Electrical and Computer Engineering, Aristotle University of \\ Thessaloniki, Thessaloniki GR-54124, Greece \\ ${ }^{\mathrm{b}}$ Centre d'Élaboration de Matériaux et d'Études Structurales, CEMES, CNRS, \\ Bô̂te Postale 94347, 31055 Toulouse Cedex 4, France
}

\begin{abstract}
Single-layer cholesteric liquid crystals exhibit a reflection coefficient which is at most 50\% for unpolarized incident light. We give theoretical and experimental evidence of single-layer polymer-stabilized cholesteric liquid crystalline structures that demonstrate hyper-reflective properties. Such original features are derived by the concurrent and randomly interlaced presence of both helicities. The fundamental properties of such structures are revealed by detailed numerical simulations based on a stochastic approach.
\end{abstract}

Key words: Cholesteric liquid crystals, photonic bandgap materials, polymer-dispersed liquid crystals, domain structures PACS: 42.70.Df, 42.70.Qs, 61.30.Eb

\section{Introduction}

The cholesteric liquid crystalline (CLC) structure is omnipresent in living matter at various scales and in various tissues, for specific or still unknown functions: condensed phases of DNA [1], cellulose matrix in plant cell walls, chitin matrix in arthropods cuticles [2], collagen network in human compact

Email address: mkriezis@auth.gr (E. E. Kriezis). 
bone [3], virus suspensions [4] or atherosclerosis disease [5]. In technology, the optical properties of the CLC phase are very attractive for numerous applications such as polarizer-free reflective displays [6], polarizers and colour filters [7], mirrorless lasing [8] or smart switchable reflective windows for the dynamical control of solar light [9]. CLCs selectively reflect light due to their helical structure $[10,11]$; when incident light propagates along the helical axis, selective reflection occurs, if the wavelength $\lambda$ is of the order of the pitch $P$ of the helix, for circularly polarized light with the same handedness as the CLC helix. At normal incidence, the central Bragg reflection wavelength is given by $\lambda_{0}=\bar{n} P$, where $\bar{n}=\left(n_{e}+n_{o}\right) / 2$ is the average refractive index, while reflection occurs within a bandwidth $\Delta \lambda=\Delta n P$, where $\Delta n=n_{e}-$ $n_{o}$. This polarization selectivity rule is a unique feature of CLCs, since a normal dielectric mirror inverses the handedness. The maximum reflectance of ambient, unpolarized, light from a CLC structure never exceeds $50 \%$ at normal incidence, as only left- or right-circularly polarized light may be reflected. Total reflectance may be achieved by forming a bilayer system where two individual cholesteric layers with the same pitch and inverse helicity sense are stacked together [12]. A multilayer solution is regularly undesired due to the optical losses at the interfaces (reflection, scattering) and the cost of implementation for applications. Recent experimental results have reported the elaboration procedure of single-layer CLC materials that are not restricted by the polarization selectivity rule, as the reflection band applies to circularly polarized light components of both helicities [13].

Photopolymerizable and crosslinkable monomers (RM257 nematic diacrylate 3.8 wt.\%, Merck Ltd.) are introduced in the volume of a CLC exhibiting a thermally induced helicity inversion; the blend consists of the nematic LC BL006 (from Merck Ltd., 71.2 wt.\%) and a chiral dopant to induce the helicity inversion (propanediol derivative, $25 \mathrm{wt} \%$ [14]). The material is introduced into a $50 \pm 10 \mu \mathrm{m}$ thick glass sandwich-cell with planar anchoring to induce Bragg planar textures. The cell is then cured with ultraviolet light $\left(0.1 \mathrm{~mW} / \mathrm{cm}^{2}\right.$ at $365 \mathrm{~nm}$ ) at a temperature for which the helix is $\mathrm{RH}$ and exhibits a pitch equal to $P_{0}$. The sample is then cooled until a temperature for which, before photopolymerization, the CLC structure was LH with a pitch close to $P_{0}$. The transmittance properties are investigated using a Perkin Elmer IR spectrophotometer coupled with a Mettler hot stage. Whatever the polarization of incident light, the reflectance of the cholesteric film goes over the limit of $50 \%$ because of the contribution of both helicities. A fraction of LC molecules is strongly bound to the polymer network and gives rise to a $\mathrm{RH}$ helical structure (template effect) and a free fraction far from the polymer surfaces forms a LH helix.

These novel experimental results may open the route to hyper-reflective display applications, for which the $50 \%$ reflectance limit is exceeded. Nevertheless, the analysis of such complex structures has not yet been theoretically 
addressed; in this context, the present study investigates the optical properties of single-layer polymer-stabilized CLC structures with material regions of both helicities, demonstrating that the existence of alternating domains of $\mathrm{RH}$ and LH material can account for an overall reflectance that exceeds the $50 \%$ limit. The optical properties of the CLC structures are studied numerically by means of the Finite-Difference Time-Domain (FDTD) method, which is capable of dealing with light propagation problems through anisotropic media $[15,16]$. Assuming normal incidence, a one dimensional algorithm is sufficient to describe the spatial variation of the CLC's dielectric tensor, while all optical power transmission coefficients presented hereinafter were calculated for the case of unpolarized incident light [17]. The accuracy of the numerical studies was verified by direct comparison of indicative examples with the $4 \times 4$ Berreman matrix method [18]; the selection of the FDTD method was due to the fact that it provides significantly faster simulation times for the cases here addressed.

\section{Optical properties of cholesteric structures comprising both he- licities}

As a starting point, the transmission spectra for two ideal structures of homogeneous CLC layers are presented in Fig. 1, for a typical liquid-crystalline compound characterized by $n_{o}=1.5, n_{e}=1.7$ and a pitch value of $P_{0}$. The first one refers to a single layer of RH material with a total thickness of $d_{1}=10 P_{0}$, while the second is a bilayer structure of total thickness $d_{2}=20 P_{0}$, divided equally between two homogeneous layers of $\mathrm{RH}$ and $\mathrm{LH}$ material. As theoretically predicted, selective reflection for the single layer rises up to $50 \%$ within the bandgap forming around the central Bragg wavelength $\lambda_{0}=0.5\left(n_{o}+n_{e}\right) P_{0}$, while in the case of the bilayer structure almost all optical power is reflected because of the accumulative effect of the two stacked layers of $\mathrm{RH}$ and $\mathrm{LH}$ helicity. It should be remarked that the optical simulations presented in this analysis account for no losses whatsoever, e.g. owing to scattering or absorption; thus, wavelength-dependent power transmission levels are attributed solely to the selective reflection properties of the CLC structures. In the case of typical polymer-stabilized CLC structures, i.e. where the photopolymerizable monomer concentration constitutes a small wt. percentage, it is pointed out that the formation of the polymer network does not significantly distort the planar structure and thus no high levels of scattering are induced [19-21,9]. In addition, when present, light scattering in such structures manifests itself primarily as a background wavelength-independent phenomenon; thus, it does not affect their reflective behaviour inside the Bragg bands, which is attributed solely to coherent reflection from the domains that develop in periodical helical configurations of the CLC material. Owing to the absence of loss mechanisms, 


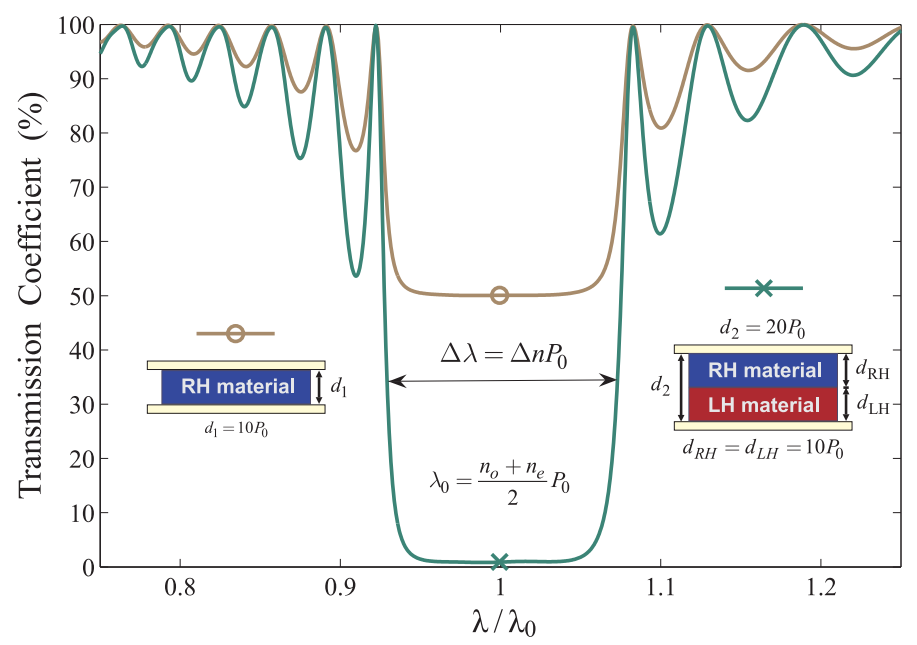

Fig. 1. (Color online) Transmittance for a single right-handed CLC layer of thickness $d_{1}=10 P_{0}$ and a bilayer structure of thickness $d_{2}=20 P_{0}$ equally divided between two stacked homogeneous layers of right- and left-handed helicity.

the reflectance spectra are simply recovered as $R(\lambda)=1-T(\lambda)$, where $R(\lambda)$ and $T(\lambda)$ are the power reflection and transmission coefficients, respectively.

Keeping the results obtained for the bilayer system as a reference, we consider next the case of CLC layers where $\mathrm{RH}$ and $\mathrm{LH}$ domains are randomly interlaced, as observed in realistic structures such as those described in [13,22]. Taking into account that the distribution of such domains within the layer cannot be described in practice by a deterministic model, it should be consistent to assess the overall optical behavior of such structures as an average of the contribution of stochastically generated one-dimensional columns/samples [16,23]. Samples are divided into a number of sublayers of fixed thickness $d_{s}$, occupied either by RH or LH material according to a probability distribution that determines the total percentage attributed to each handedness. The transmittance of each sample is calculated independently and the total transmission spectrum is derived by averaging over a sufficient number of samples, generated according to a normal distribution.

Figure 2 presents the transmittance spectra, each corresponding to 500 randomly generated samples of fixed total thickness $d=20 P_{0}$, and various sublayer thickness values $\left(d_{s}=2 P_{0}, P_{0}, 0.5 P_{0}\right.$, and $\left.0.25 P_{0}\right)$, where the ratio between $\mathrm{RH} / \mathrm{LH}$ regions is maintained around unity in all cases. It can be observed that independently of the value of $d_{s}$, total reflection around $\lambda_{0}$ is close to $100 \%$, indicating that despite the stochastic pattern according to which they were generated, such disordered structures of alternating sublayers of $\mathrm{RH}$ and LH material may provide almost total reflectance within a significant wavelength window around $\lambda_{0}$. In contrast, the reflective efficiency close to the edges of the reference bandgap region progressively drops while $d_{s}$ obtains lower values, as revealed by a direct comparison with the dotted reference 


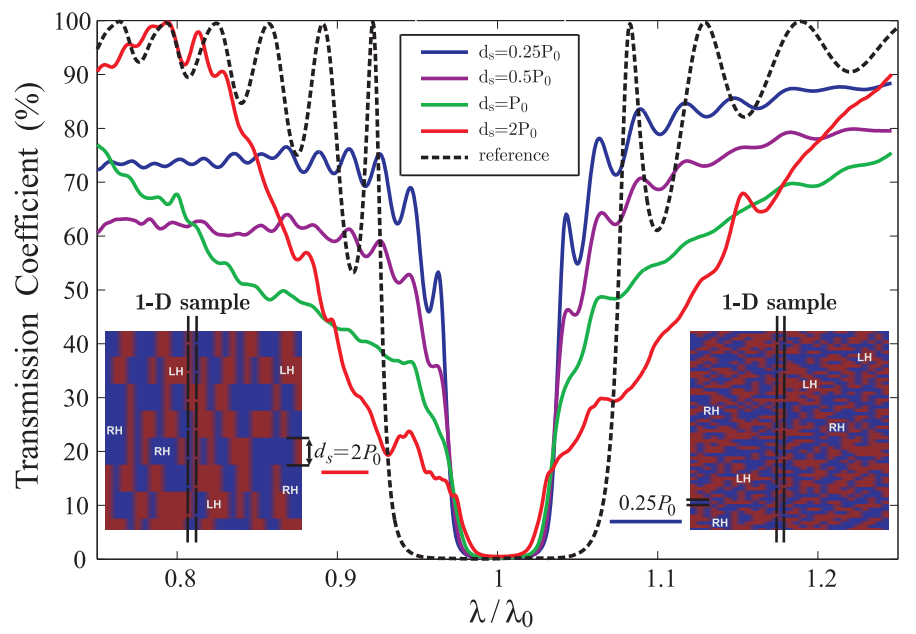

Fig. 2. (Color online) Transmittance for a monolayer of thickness $d=20 P_{0}$ distributed equally among randomly located sublayers of $\mathrm{RH}$ and $\mathrm{LH}$ material for various values of sublayer thickness $d_{s}$. The dotted reference curve corresponds to the bilayer system of Fig. 1. Insets show cross-sectional views of patterns formed by the generated stochastic samples for $d_{s}=2 P_{0}$ and $d_{s}=0.25 P_{0}$. (Color code LH:red, RH:blue)

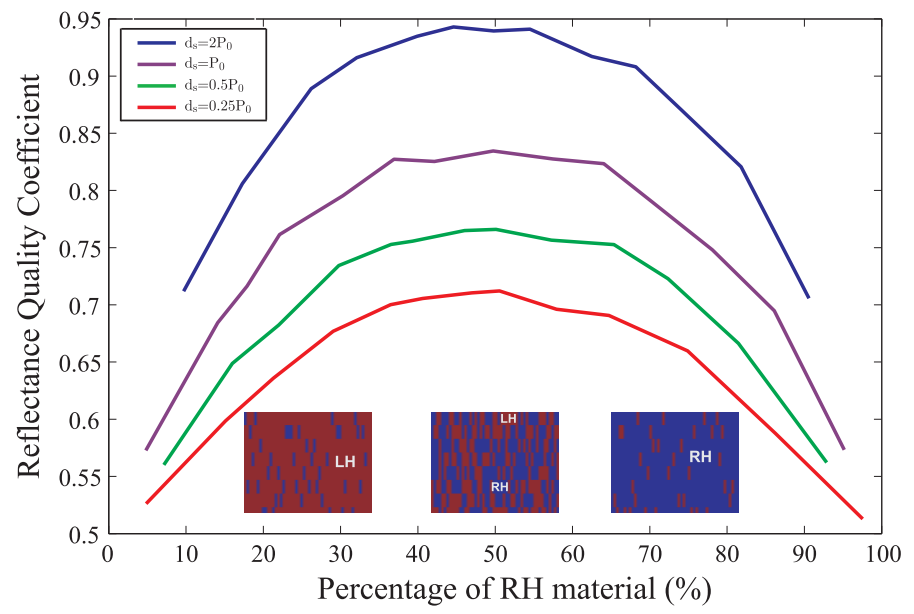

Fig. 3. (Color online) Reflectance quality coefficient for monolayers of thickness $d=20 P_{0}$ for various values of sublayer thickness $d_{s}$ and distributions between $\mathrm{RH}$ and LH material. Maximum reflectivity is observed for $d_{s}=2 P_{0}$ and equal percentages of $\mathrm{RH}$ and $\mathrm{LH}$ regions. Inset shows typical distributions of the material for increasing percentages of RH material.

curve of the bilayer system of Fig. 1.

Figure 3 provides an overall estimate of the reflective efficiency of the investigated structures, via the assessment for varied values of both $d_{s}$ and the percentages attributed to $\mathrm{RH}$ and $\mathrm{LH}$ regions of a reflectance quality coeffi- 


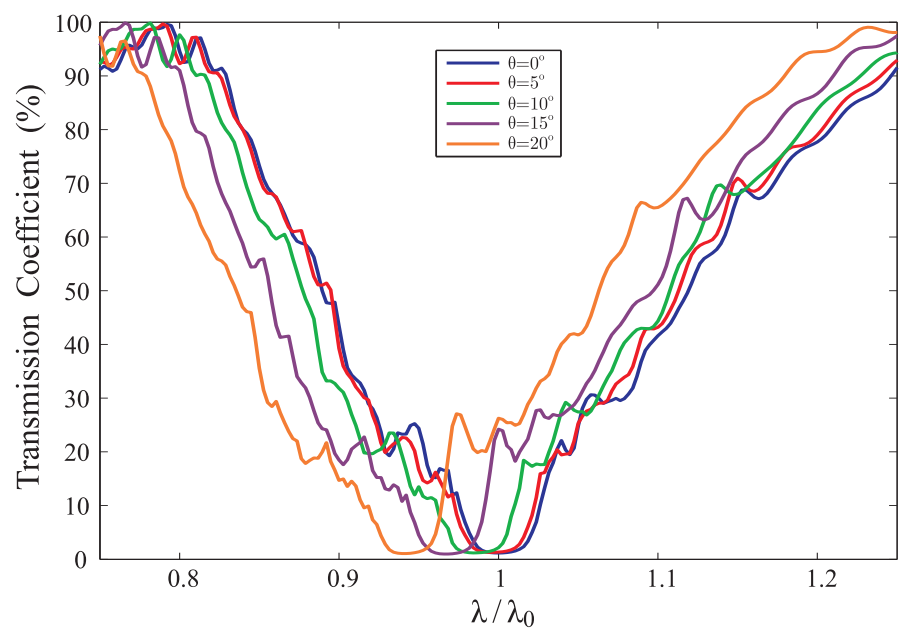

Fig. 4. (Color online) Transmittance for a monolayer of thickness $d=20 P_{0}$ distributed equally among randomly located sublayers of $\mathrm{RH}$ and $\mathrm{LH}$ material for $d_{s}=2 P_{0}$ and various angles of incidence $\theta$. A blue-shift is observed as $\theta$ obtains higher values, while the shape of the bandgap transmission spectra remains otherwise practically unaffected.

cient

$$
\widetilde{R} \equiv 1-\frac{1}{\Delta \lambda} \int_{\lambda_{1}}^{\lambda_{2}} T(\lambda) d \lambda, \quad \Delta \lambda=\lambda_{2}-\lambda_{1}
$$

which represents the average power reflection coefficient within the bandgap wavelength window $\left(\lambda_{1}=n_{o} P_{0}, \lambda_{2}=n_{e} P_{0}\right)$, and $T(\lambda)$ is the power transmission coefficient function, defined as in [17]. It is shown that the reflective performance is enhanced when the percentage of both regions is approximately equal and when the thickness of the sublayer $d_{s}$ is increased, which corresponds to structures where $\mathrm{RH}$ and $\mathrm{LH}$ regions exhibit higher homogeneity. In any case, it is clearly demonstrated that CLC layers with randomly distributed areas of alternating senses of helicity are capable of providing enhanced reflectance that exceeds the $50 \%$ limit. It is emphasized that this particular study has dealt only with the normal incidence case, both from the experimental as well as from the theoretical point of view. In the case of oblique incidence it is anticipated that the reflection band will experience a blue-shift, while the reflection band $\Delta \lambda$ should remain practically unaffected [24]. As an indicative example, Fig. 4 shows the transmission spectra corresponding to the $d_{s}=2 P_{0}$ case of the structure investigated in Fig. 2, obtained for various angles of incidence by the Berreman $4 \times 4$ matrix method (still summing over 500 randomly generated samples); apart from the expected blue-shift as $\theta$ obtains higher values, the shape of the bandgap reflection windows is not noticeably modified. 


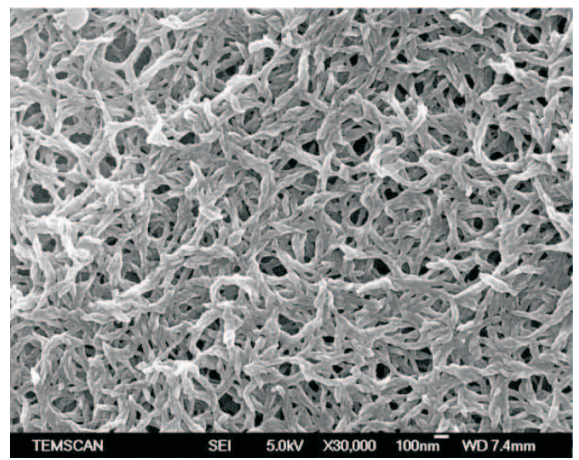

(a)

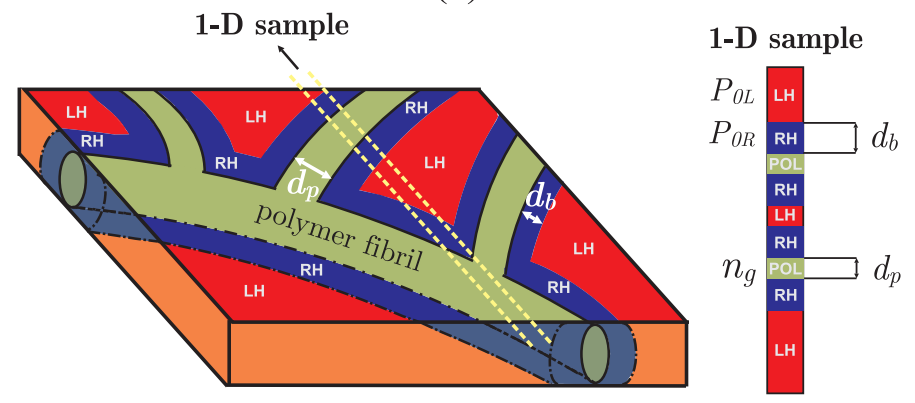

(b)

Fig. 5. (Color online) (a) SEM image showing a typical network formed in polymer-stabilized CLC structures after the removal of the LC material [25], and (b) material structure and definition of the related parameters used in the model adopted for the numerical study: $n_{g}$ denotes the polymer's refractive index, $d_{p}$ the mean thickness of the polymer fibrils, $d_{b}$ the mean thickness of $\mathrm{RH}$ material bound to the polymer network, and $P_{0 L}, P_{0 R}$ the pitch value for the $\mathrm{LH}$ and $\mathrm{RH}$ regions, respectively.

\section{Optical properties of polymer-stabilized cholesteric structures comprising both helicities}

The theoretical model under consideration is next generalized to the case of realistic polymer-network CLC structures, such as the one depicted in the Scanning Electron Microscopy (SEM) image of Fig. 5(a), according to the schematic layout and related parameters shown in Fig. 5(b). The 1-D columns/samples are constructed by randomly placing polymer layers of refractive index $n_{p}$ and variable thickness around a mean value of $d_{p}$. Attached to the polymer network, the RH bound material is assumed to extend for a mean thickness $d_{b}$, while the rest of the space is occupied by the free LH bulk material. The pitch value for the LH region is fixed at $P_{0 L}=P_{0}$, whereas in the $\mathrm{RH}$ region some shrinkage of the pitch value is allowed $\left(P_{\min } \leq P_{0 R} \leq P_{0}\right)$ in agreement with experimental findings. Indeed, after photopolymerization the pitch value is slightly decreased as a result of a volume shrinkage effect related to the polymer network formation in the LC matrix [22]; such a behaviour was 


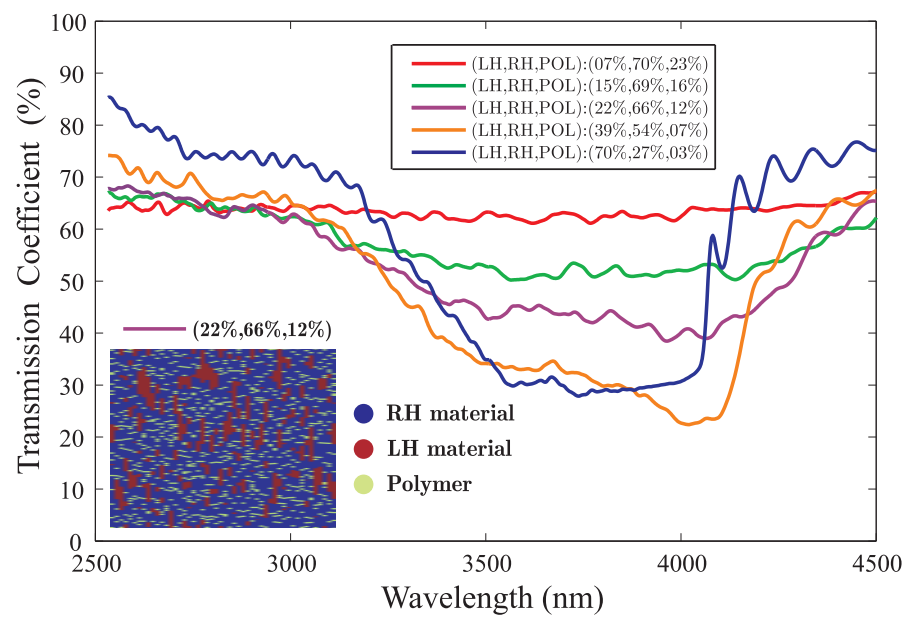

Fig. 6. (Color online) Transmittance for a polymer-stabilized CLC monolayer of thickness $d=50 \mu \mathrm{m}$, for different distributions of $\mathrm{RH}, \mathrm{LH}$, and polymer materials. Structural and material parameters (as defined in Fig. 5): $n_{g}=1.5$, $d_{p}=150 \sim 250 \mathrm{~nm}, d_{b}=1.5 \sim 2.2 \mu \mathrm{m}, P_{0 L}=P_{0}=2.375 \mu \mathrm{m}$, and $P_{0 R}=0.93 P_{0} \sim P_{0}$. As an example, the inset shows the pattern formed by the generated stochastic samples for a $\mathrm{LH} / \mathrm{RH} /$ polymer ratio of $22 \% / 66 \% / 12 \%$.

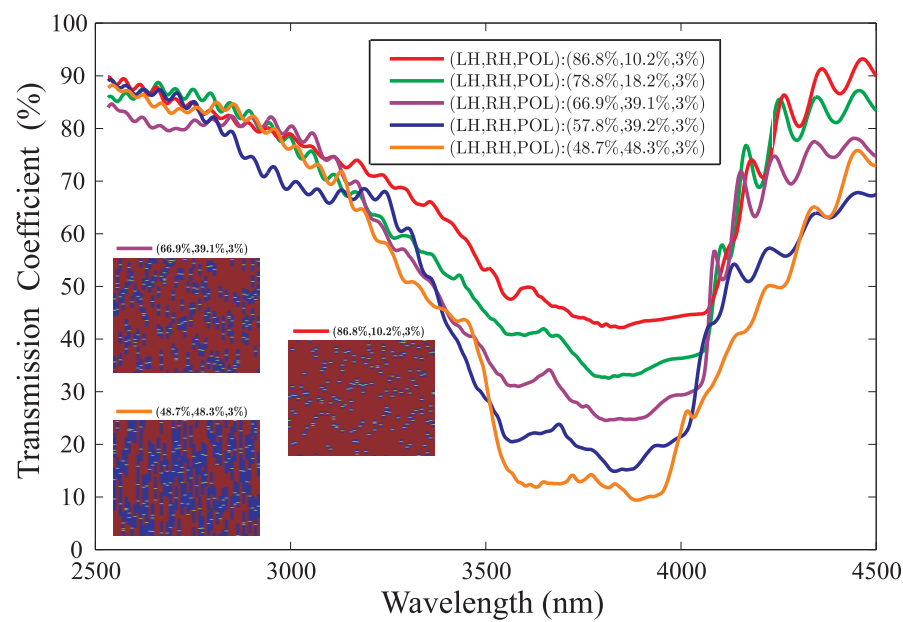

Fig. 7. (Color online) Transmittance for a polymer-stabilized CLC with fixed polymer ratio $(3 \%)$ and values of $d_{b}$ ranging from $0.5 \sim 0.7 \mu m$ (red curve) to $4 \sim 4.2 \mu m$ (orange curve). Other parameters as in Fig. 6. Insets show the pattern formed by the stochastically generated samples for three ratios of $\mathrm{LH} / \mathrm{RH} /$ polymer distributions.

also observed during the formation of ordered polymer networks with a helical structure [26].

Figure 6 shows the transmission spectra obtained for polymer-stabilized CLCs designed for mid-infrared reflection [13,22], for pitch values $P_{0 L}=P_{0}=$ $2.375 \mu \mathrm{m}, 0.93 P_{0} \leq P_{0 R} \leq P_{0}$, polymer index $n_{g}=1.5$, polymer strand thickness $d_{p}=150 \sim 250 \mathrm{~nm}$ and bound RH material thickness $d_{b}=1.5 \sim 2.5 \mu \mathrm{m}$. The overall distribution among $\mathrm{RH}, \mathrm{LH}$ and polymer regions is controlled by adjusting the percentage of the polymer in the samples. It can be noticed 


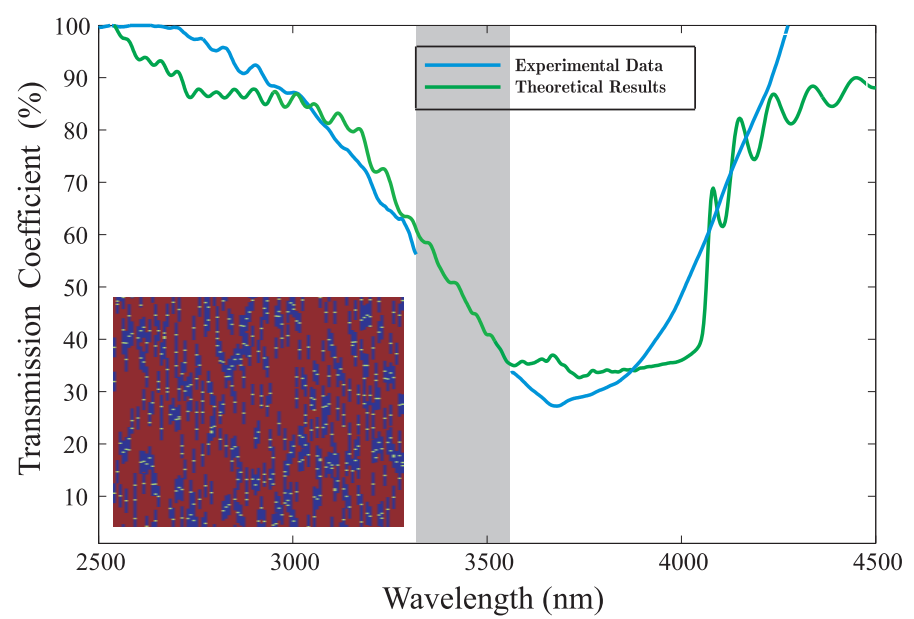

Fig. 8. (Color online) Comparison between experimental data [13] and theoretical calculations for a polymer-stabilized CLC structure of thickness $d=50 \mu \mathrm{m}$ and a ratio of $\mathrm{LH} / \mathrm{RH} /$ polymer regions equal to $70 \% / 27 \% / 3 \%$. (The shadowed area marks the chemical absorption bands.)

that as the polymer network becomes denser (more than 10\%) and the $\mathrm{RH}$ material dominates, the bandgap progressively vanishes and the structure's spectral response is flat, implying a highly scattering state; in contrast, for polymer percentage values of a few percent enhanced reflectivity is demonstrated, which exceeds the $50 \%$ limit. These results are in agreement with experimental observations; a polymer concentration threshold exists, above which the free fraction of LC molecules is drastically confined in the dense network resulting, thus, in a polydomain structure that induces high levels of light scattering $[22,25]$.

Assuming a fixed value (3\%) for the percentage of the polymer network, which may be directly correlated with experimentally studied structures [9,27], the ratio between $\mathrm{RH}$, LH regions may be associated to the exact value of the thickness $d_{b}$ of the $\mathrm{RH}$ material bound to the polymer network. Figure 7 investigates the transmission spectra obtained for value distributions of $d_{b}$ ranging from $0.5 \sim 0.7 \mu m$ (red curve) to $4 \sim 4.2 \mu m$ (orange curve). It is once more demonstrated that for approximately equal percentages of $\mathrm{RH}$ and LH material, the reflectivity of the investigated polymer-network CLC structures exceeds $50 \%$, obtaining values as high as $\sim 90 \%$ for a $\mathrm{LH} / \mathrm{RH}$ ratio of $48.7 \% / 48.3 \%$.

As a final elucidating example, Fig. 8 shows a comparison between experimental data for a polymer-stabilized CLC structure obtained as discussed in $[13,22]$ and theoretical results based on the described model. The experiments were conducted for the case of normal incidence, while light was also collected at the output of the sample along the normal direction; numerical simulations were performed according to the following set of parameters: $d=50 \mu \mathrm{m}$, $n_{g}=1.5, d_{p}=150 \sim 250 \mathrm{~nm}, d_{b}=1.5 \sim 2.2 \mu \mathrm{m}, P_{0 L}=P_{0}=2.375 \mu \mathrm{m}$, 
and $0.93 P_{0} \leq P_{0 R} \leq P_{0}$ and $\mathrm{RH} / \mathrm{LH} /$ polymer ratio equal to $70 \% / 27 \% / 3 \%$. In order to allow for a direct comparison, the theoretical curve was normalized according to the normalization conditions of the experimental findings, i.e. assuming $100 \%$ transmittance at the low wavelength limit. Taking into account the stochastic nature of the formation of such structures, the proposed numerical model reproduces with very high accuracy the experimental curve.

\section{Conclusions}

In conclusion, we have presented a theoretical model that investigates in detail the optical properties of recently proposed hyperreflective polymer-CLC gels based on thermally induced helicity inversion and provides significant insight to questions regarding their structural morphology and properties. Results demonstrate that such structures are particularly promising in terms of the realization of CLC films with exceptionally large fluxes of reflected light that might prove indispensable in polarizer-free reflective displays, tunable glazing panels, smart windows in buildings or vehicles, or temperature-control devices.

\section{Acknowledgements}

The authors acknowledge the financial support of the Greek General Secretariat of Research and Technology.

\section{References}

[1] F. Livolant, A. Leforestier, Prog. Polym. Sci. 21 (1996) 1115.

[2] A. C. Neville, Biology of Fibrous Composites: Development Beyond the Cell, Cambridge Univ. Press, 1993.

[3] M.-M. Giraud-Guille, Calcif. Tissue Int. 42 (1988) 167.

[4] Z. Dogić, S. Fraden, Langmuir 16 (2000) 7820.

[5] D. M. Small, J. Colloid. Interf. Sci. 58 (1977) 581.

[6] Y. Huang, Y. Zhou, S.-T. Wu, Opt. Express 15 (2007) 6414.

[7] N. Y. H. et al., Nat. Mater. 7 (2008) 43.

[8] W. Cão, A. Muñoz, P. Palffy-Muhoray, B. Taheri, Nat. Mater. 1 (2002) 111. 
[9] M. Mitov, E. Nouvet, N. Dessaud, Eur. Phys. J. E 15 (2004) 413.

[10] P. de Gennes, J. Prost, The Physics of Liquid Crystals, Clarendon Press: Oxford, 1993.

[11] I. J. Hodgkinson, Q. H. Wu, M. Arnold, M. W. McCall, A. Lakhtakia, Opt. Commun. 210 (2002) 201.

[12] D. M. Makow, Appl. Opt. 19 (1980) 1274.

[13] M. Mitov, N. Dessaud, Nat. Mater. 5 (2006) 361.

[14] G. Heppke, D. Lötzch, F. Oestreicher, Z. Naturforsch. 42a (1987) 279.

[15] E. Kriezis, S. Elston, Opt. Commun. 177 (2000) 69.

[16] D. C. Zografopoulos, E. E. Kriezis, M. Mitov, C. Binet, Phys. Rev. E 73.

[17] S. Stallinga, J. Appl. Phys. 85 (1999) 3023.

[18] D. W. Berreman, J. Opt. Soc. Am. 62 (1972) 502.

[19] R. A. M. Hikmet, B. H. Zwerwer, Liq. Cryst. 12 (1992) 319.

[20] I. Dierking, L. L. Kosbar, A. Afzali-Ardakani, A. C. Lowe, G. A. Held, J. Appl. Phys. 81 (1997) 3007.

[21] C. Binet, M. Mitov, M. Mauzac, J. Appl. Phys. 90 (2001) 1730.

[22] M. Mitov, N. Dessaud, Liq. Cryst. 34 (2007) 183.

[23] C. Bohley, T. Scharf, Opt. Commun. 214 (2002) 193.

[24] Q. Hong, T. Wu, S.-T. Wu, Liq. Cryst. 30 (2003) 367.

[25] S. Relaix, Ph.D. thesis, Université Toulouse III - Paul Sabatier, UFR PCA (2007).

[26] D. J. Broer, I. Heynderickx, Macromol. 23 (1990) 2474.

[27] R.-Q. Ma, D.-K. Yang, Phys. Rev. E 61 (2000) 1567. 